\title{
COVID-19 in the Context of Inborn Errors of Immunity: a Case Series of 31 Patients from Mexico
}

\author{
Lina M. Castano-Jaramillo ${ }^{1}$. Marco Antonio Yamazaki-Nakashimada ${ }^{1} \cdot$ Patricia M. O'Farrill-Romanillos ${ }^{2}$. \\ David Muzquiz Zermeño ${ }^{3}$. Selma C. Scheffler Mendoza ${ }^{1}$ Edna Venegas Montoya ${ }^{3}$. Jorge Alberto García Campos ${ }^{4}$. \\ Luz María Sánchez-Sánchez ${ }^{5}$. Luisa B. Gámez González ${ }^{6}$. Jesús Moisés Ramírez López ${ }^{7}$. \\ Juan Carlos Bustamante Ogando ${ }^{8}$. Estefanía Vásquez-Echeverri ${ }^{8} \cdot$ Edgar Alejandro Medina Torres $^{8}$. \\ Gabriela Lopez-Herrera ${ }^{8} \cdot$ Lizbeth Blancas Galicia $^{8} \cdot$ Laura Berrón Ruiz $^{8}$. Aidé Tamara Staines-Boone ${ }^{3}$. \\ Sara Elva Espinosa-Padilla ${ }^{8} \cdot$ Nora Hilda Segura Mendez $^{2} \cdot$ Saul O. Lugo Reyes $^{8}$
}

Received: 16 March 2021 / Accepted: 25 May 2021 / Published online: 10 June 2021

(C) The Author(s), under exclusive licence to Springer Science+Business Media, LLC, part of Springer Nature 2021

\begin{abstract}
Highlights
1. What is already known about this topic? SARS-CoV-2 causes asymptomatic or mild infection in about $80 \%$ of humans, while an excessive immune response has killed millions. Differential susceptibility and risk factors became a concern early in the pandemic. Several monogenic defects that involve innate viral sensors or affect interferon response pathways, as well as autoantibodies against type 1 interferons, have been identified in $14 \%$ of patients with life-threatening COVID-19. The impact of the novel betacoronavirus infection in patients with known inborn errors of immunity is less clear. Case series and reports from different countries have suggested a minor impact or even a potential protective effect of the IEI for some patients.

2. What does this article add to our knowledge? We describe findings and outcomes of COVID-19 in 31 pediatric and adult patients with known IEI from Mexico, $84 \%$ of whom survived. Pediatric patients had a higher hospitalization rate. Inpatient mortality was $40 \%$, and ICU mortality was $63 \%$. Six patients died of secondary bacterial infection or uncontrolled systemic inflammation, but not from overwhelming viral infection. One patient with an autoinflammatory disorder under treatment with anakinra had a catastrophic clinical course. Eighty percent of patients received IVIG as part of their treatment for acute SARSCoV-2 infection.

3. How does this study impact current management guidelines? We recommend continued and/or high-dose IVIG in patients with known IEI seeking care for COVID-19. Patients with autoinflammatory disorders, especially those with inflammasome dysregulation, should probably take extreme measures to prevent exposure, while doctors taking care of SARS-CoV-2 infected patients with immune deficiencies must do everything they can to prevent secondary bacterial infections. The high survival of patients with COVID-19 in the context of inborn errors of immunity worldwide (over 80\%) might be the result of patientphysician awareness and special care.
\end{abstract}

Extended author information available on the last page of the article 


\section{Abstract}

Introduction Patients with inborn errors of immunity (IEI) have a compromised or inappropriate immune response. Although they might be considered a high-risk group for severe SARS-CoV-2 infection, the reported impact of COVID-19 in these patients has been reassuring, while the differential susceptibility of distinct types of IEI remains unclear.

Objective We aimed to describe the findings and outcomes of our known patients with IEI who were diagnosed with COVID-19.

Methods In a retrospective study from March 2020 to February 2021, four centers in Mexico collected clinical, laboratory, and genetic data from pediatric and adult patients with known diagnoses of IEI who presented with COVID-19, based on compatible symptoms and positive SARS-CoV-2 testing or known household exposure.

Results We report 31 patients with known IEI from Mexico who presented with SARS-CoV-2 infection. Seventy-four percent were male, $52 \%$ were pediatric, and $81 \%$ survived. Their ages ranged from 5 months to 56 years, with a median of 17 years. Sixty-five percent had predominant antibody deficiencies, $48 \%$ were hospitalized, and $26 \%$ required ICU. Pediatric patients had a higher hospital admission rate than adults. Inpatient mortality was $40 \%$, and ICU mortality rate was $63 \%$. Forty-eight percent developed pneumonia, while $36 \%$ had evidence of hyperinflammation (4 adults and 7 children). Predominant laboratory features were lymphopenia and thrombocytopenia, seen in 70 and $44 \%$ of patients, respectively. The serum D-dimer median value was $2.6(0.5-20.6) \mu \mathrm{g} / \mathrm{mL}$, and the median highest ferritin value was $1015(32-10,303) \mathrm{ng} / \mathrm{mL}$. Intravenous immunoglobulin was used in $80 \%$ of patients. Other treatments included macrolides (39\%) and corticosteroids (29\%). Six patients died from secondary infection or uncontrolled systemic inflammation.

Discussion Although impaired immunity due to IEI may be a predisposing factor for severe COVID-19, most of our patients with IEI who acquired the SARS-CoV-2 infection developed a well-tolerated infection and survived, as have more than $80 \%$ of worldwide reported patients to date. An impaired immune or inflammatory response may be a predisposing factor for some and a protective factor for others. A systematic review of the literature could help identify those patients at risk of severe disease and complications. Healthcare-associated infections should be aggressively prevented.

Keywords Primary Immunodeficiency Diseases · COVID-19 $\cdot$ SARS-CoV-2 $\cdot$ Inborn errors of immunity $\cdot$ Immune deficiencies $\cdot$ Case series $\cdot$ Mortality $\cdot$ MIS-C/PIMS $\cdot$ Hyperinflammation $\cdot$ Mexico

\section{Introduction}

The current SARS-CoV-2 pandemic has caused over 2.6 million deaths after more than 117 million people were infected worldwide. In Mexico, more than 2.1 million confirmed cases have left over 190,000 deaths after 12 months [1]. The novel betacoronavirus zoonotic spillover spread swiftly around the world in a few months, causing asymptomatic or mild disease in about $80 \%$ of patients. Differential susceptibility became an early concern, especially among patients, clinicians, and researchers living with, treating, or studying, immune deficiencies.

The main risk factors for severe SARS-CoV-2 infection, inflammatory complications, and death are old age, obesity, and male sex [2,3]. Pre-existing diseases related to "inflammaging," such as diabetes mellitus, hypertension, and chronic heart or lung disease, also predict a poor outcome [3, 4]. Strikingly, a considerable number of patients without any known risk factor have also developed critical respiratory failure, neurologic or systemic inflammatory complications, and death. An overzealous inflammatory response to the coronavirus causes more damage than the viral infection per se.

In at least $14 \%$ of patients with life-threatening COVID-19, autoantibodies against type 1 interferons, or pathogenic variants in genes involved in innate immune sensing or interferon viral response, have been identified $[5,6]$. Increased IL- $1 \beta$, IL-6, and TNF- $\alpha$ serum concentrations, and NLRP3 inflammasome activation, have been found and are known to play a role in the pathophysiology of hospitalized patients with acute respiratory distress and neurologic or systemic inflammatory response ("brain fog," "cytokine storm," "Kawasaki-like," or multisystem inflammatory syndrome) $[5,7,8]$.

Inborn errors of immunity (IEI) are a group of more than 450 rare congenital diseases with a wide clinical spectrum of increased susceptibility to infections, inflammation, atopy, autoimmunity, and/or cancer [9]. The study of patients with COVID-19 in the context of known IEI, with varying immune component deficiencies, may further inform our understanding of the virushost interactions and help dissect our immune system response to the virus.

\section{Objective}

We aimed to describe the findings and outcomes of our known patients with IEI who were diagnosed with COVID-19. 


\section{Methods}

In a multicenter retrospective study, we collected clinical, laboratory, and genetic data from patients with known diagnoses of IEI who presented with SARS-CoV-2 infection during the first 12 months of the pandemic in Mexico. Two centers in Mexico City (one for adults, one for pediatric patients), one in Monterrey, and one in Chihuahua, contributed with information.

Previously diagnosed patients (in accordance with current diagnostic guidelines) were included by their clinicians, immunologists at referral centers for IEI. SARS-CoV-2 infection was diagnosed when patients with compatible symptoms had a positive real-time reverse transcriptionpolymerase chain reaction (RT-PCR) test, a positive rapid colorimetric assay, a positive antibody test during or after the acute disease, and/or, patients who were close contacts of confirmed COVID-19 cases (household family members).

IBM SPSS Statistics for Windows, Version 26.0 (Armonk, NY) was used for statistical analysis. Qualitative data were reported as frequencies and percentages. Quantitative data were described as median, minimum, and maximum values, assuming a non-normal data distribution given the small number and heterogeneity of the patients. Nonparametric Mann-Whitney U-test was used to compare quantitative data, and Fisher's exact test was used to analyze categorical variables. A p-value $<0.05$ was considered statistically significant.

\section{Results}

Thirty-one patients with COVID-19 in the context of an IEI were identified and followed from March 2020 to February 2021, at four centers in Mexico. Most patients (74\%) were male, and almost two-thirds (20 patients, $65 \%$ ) had predominantly antibody deficiencies, group III in the International Union of Immunological Societies classification [9]. Five patients had phagocyte defects, three a combined immunodeficiency (two syndromic), one a disease of immune dysregulation, one an autoinflammatory disorder, and one a phenocopy of a primary immune deficiency (Good syndrome). Sixteen patients $(52 \%)$ had confirmed genetic diagnoses. Their ages ranged from 5 months to 56 years, with a median of 17 years. Sixteen (52\%) of the patients in our series were pediatric. Three of the patients (P2, P3, P25) had been previously included in an international report of COVID-19 in IEI [10].

Overall, $87 \%$ of the patients had preexisting comorbidities. In 58\% there was a history of chronic lung disease, bronchiectasis, asthma, or fungal or mycobacterial lung infection. In 23\% there was history of autoimmune disease, mainly cytopenias. When explored, however, differences in hospitalization, ICU admission, or outcome were not statistically significant.
For SARS-CoV-2 confirmation, multiple methods were employed. Real-time reverse transcription-polymerase chain reaction (RT-PCR) was performed in 28 patients, 24 of whom had a positive result $(86 \%)$. Of the 4 patients with negative RT-PCR, three were diagnosed based on close contact with confirmed cases and compatible symptoms, and one case was diagnosed through specific IgM for SARS-CoV-2. Of the remaining cases, one was diagnosed through a positive rapid antigen test, one based on close contact and symptoms, and one case by positive $\operatorname{IgG}$ for SARS-CoV-2. Specific IgG tests for SARS-CoV-2 were performed in 5 patients, and only 2 were positive; (one had Chronic granulomatous disease and one an Autoinflammatory disorder). Three patients had a negative $\operatorname{IgG}$ despite having previous positive RT-PCR results; these patients had either combined $(A R P C 1 B, C D 3 D)$ or predominantly antibody defects (SAD).

Almost half of the patients (48\%) were hospitalized, eight required intensive care (26\%), and six died (19\%). Fifteen of 31 patients developed COVID-19 pneumonia (48\%), and 11 of $31(36 \%)$ had evidence of hyperinflammation or MIS-C (4 adults and 7 children). Table 1 and 2 summarize the patients' clinical and laboratory characteristics. Table 3 highlights the most relevant results, and Table 4 compares hospital and ICU admittance in children and adults.

Of the 15 patients who required hospital admission 6 died, for an inpatient mortality of $40 \%$, while 5 out of the 8 patients who required intensive care died (63\%). When comparing patients with XLA and CVID (7 versus 11), no significant differences were found, neither in hospital or ICU admission, nor in mortality. The six patients that died had evidence of hyperinflammation; 2 out of the 4 pediatrics deaths were secondary to MIS-C. Mortality in patients with hyperinflammation was $55 \%$. Hyperinflammation was associated with a fatal outcome, with an OR of 2.2 (95\% CI 1.2-4.2), $p=0.001$.

The following is a short depiction of the deceased patients: P3, a 3-year-old male with WAS post-HSCT who died due to uncontrolled CMV infection; P7, a 13-year-old male with XLA who died of pulmonary bleeding in the context of secondary hemophagocytic syndrome; P19, a 38-year-old male with XLA who died from a healthcareassociated infection; P26, a 16-year-old male with CGD who died from uncontrolled MIS-C; P30, a 12-year-old male with an autoinflammatory syndrome who died from rapidly progressive MIS-C; and P31, a 51-year-old male with Good syndrome who died from a healthcare-associated infection.

Acute-phase reactants are rarely assessed in ambulatory patients. Only in 8 patients was the erythrocyte sedimentation rate (ESR) measured, 7 of whom were hospitalized; the median ESR was 43 (24-72) $\mathrm{mm}$ per hour (Reference values $<15-20 \mathrm{~mm} / \mathrm{h}$ in patients under 50 years and $<20-30 \mathrm{~mm} / \mathrm{h}$ in patients over 50 years[11]), and there 


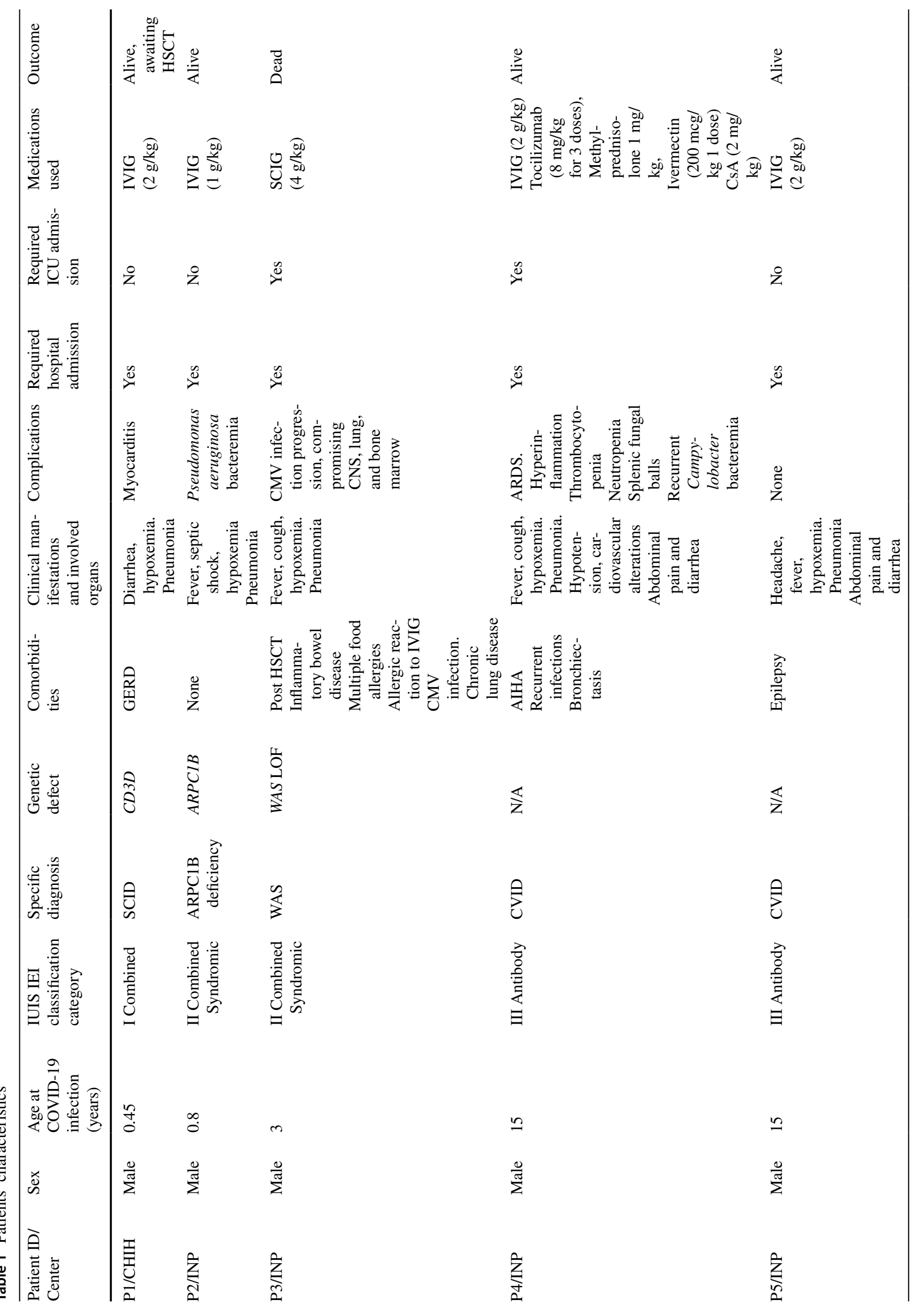




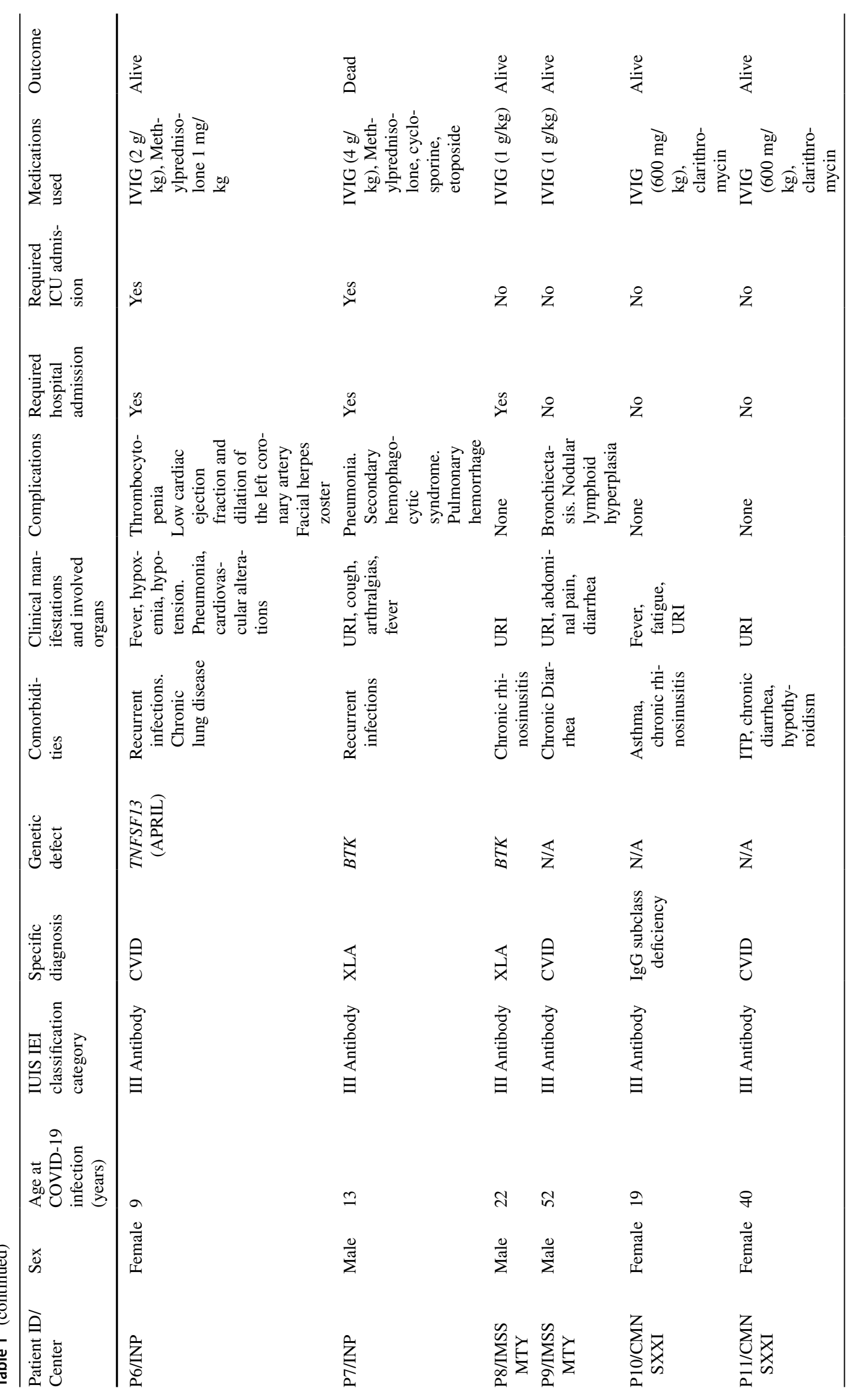


1468

Journal of Clinical Immunology (2021) 41:1463-1478

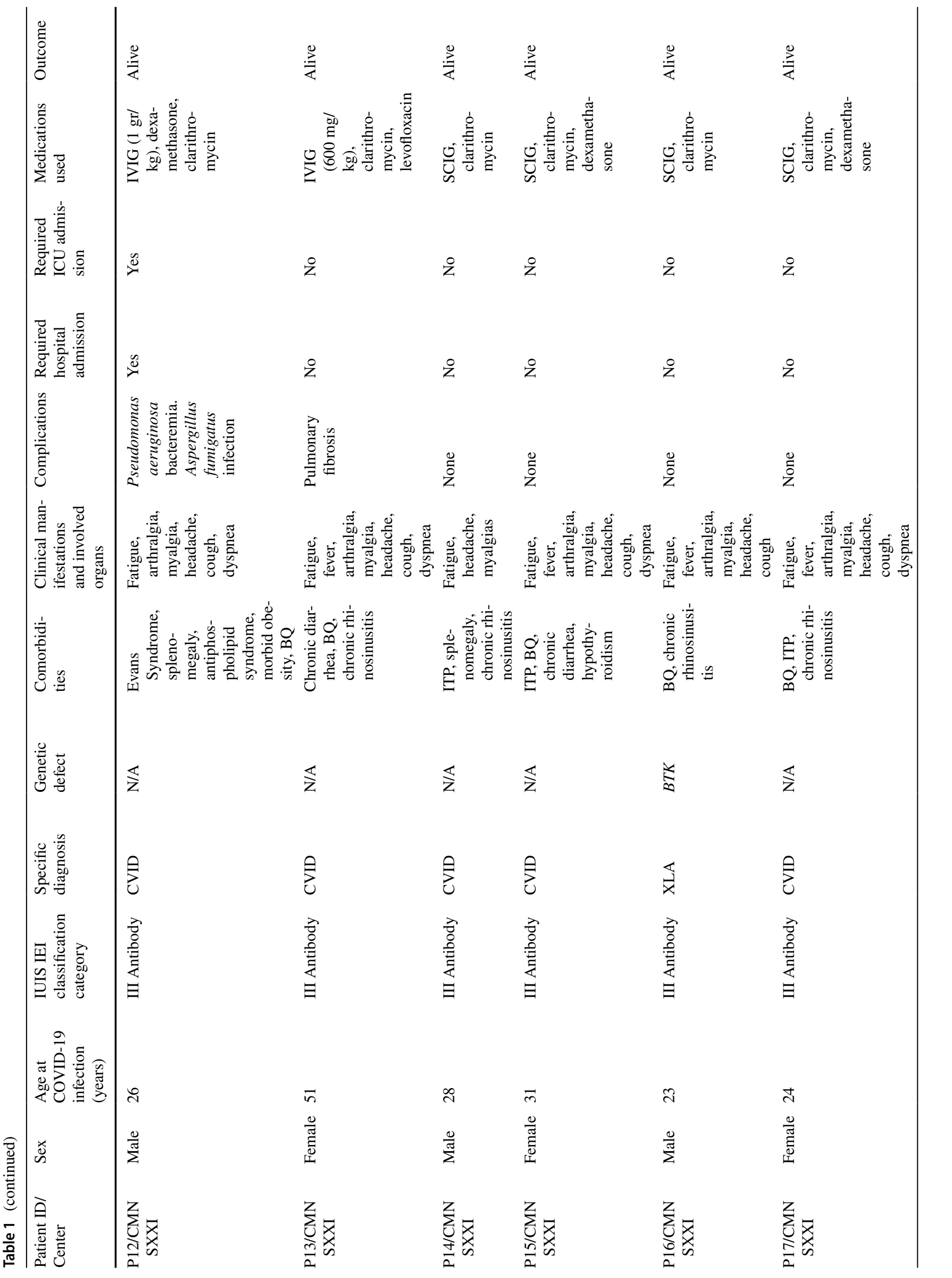

Springer 
Journal of Clinical Immunology (2021) 41:1463-1478

1469

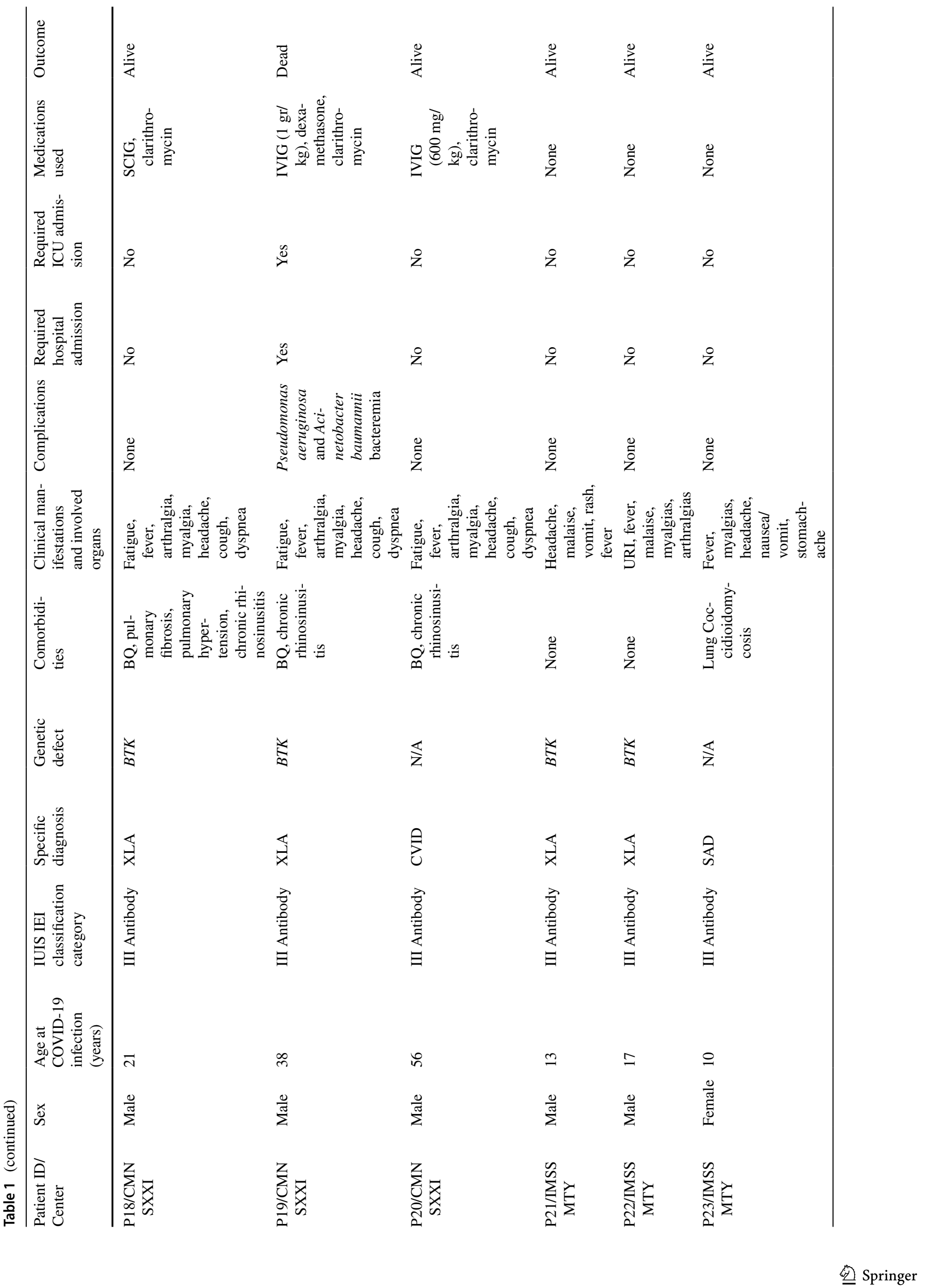




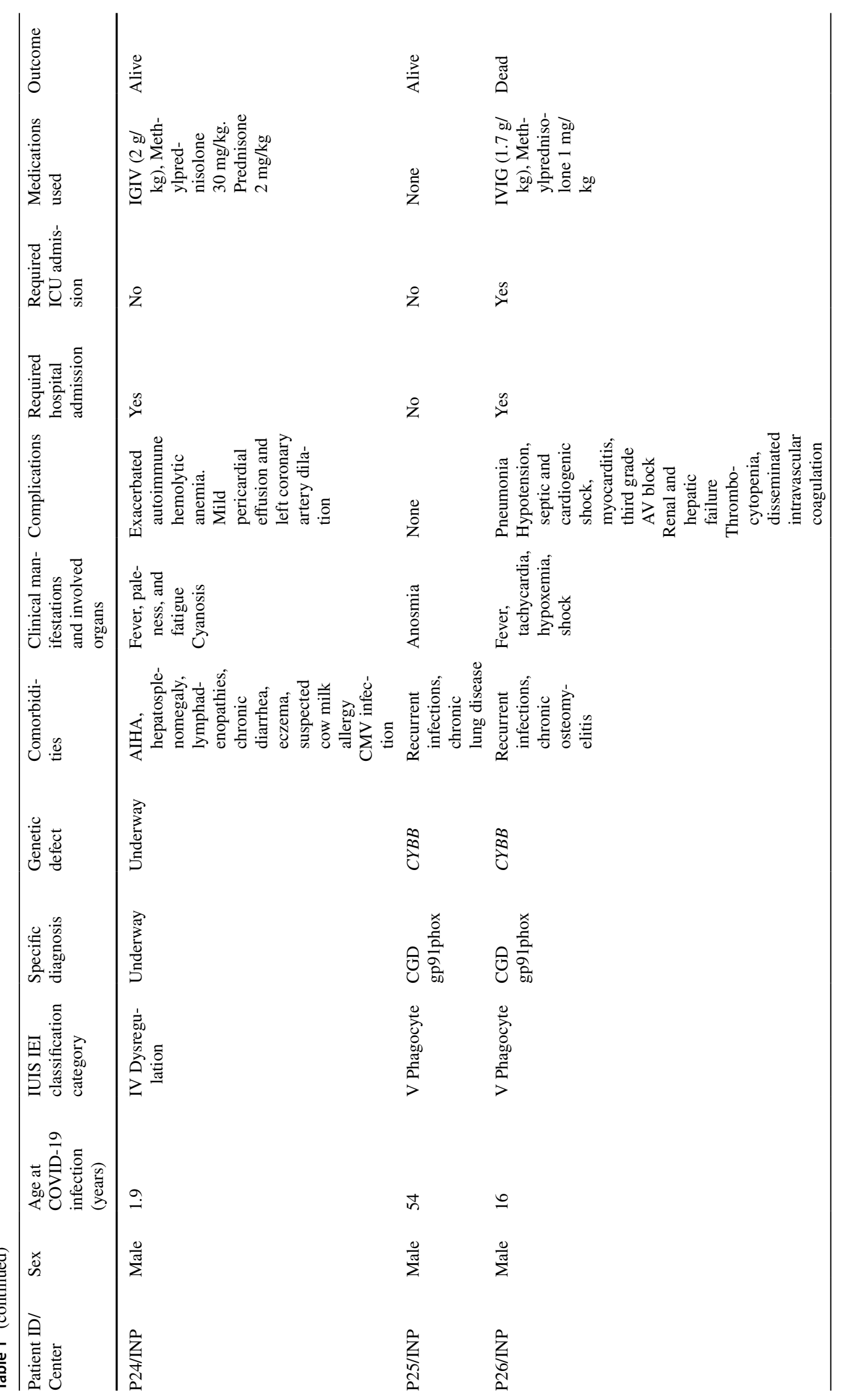




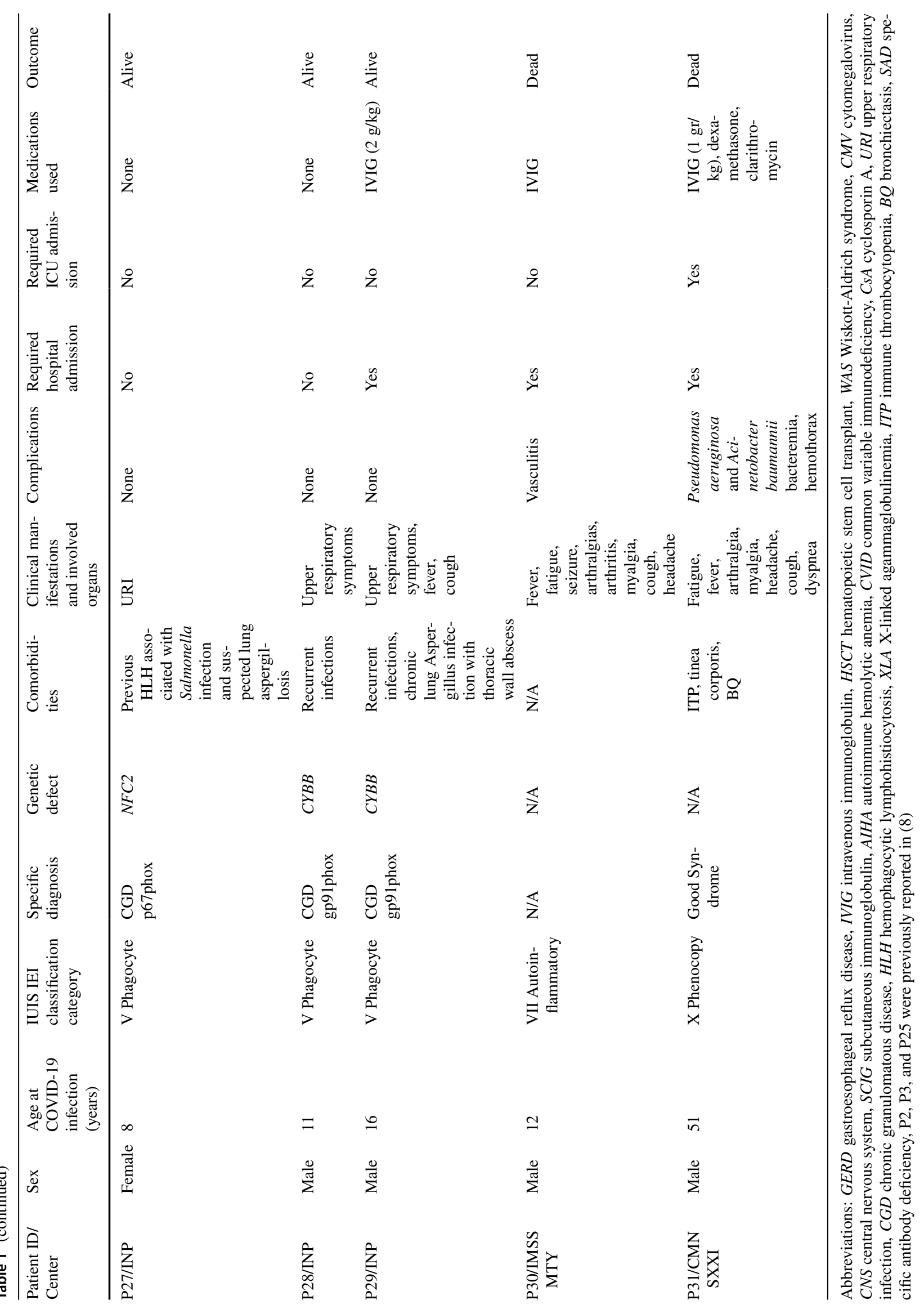




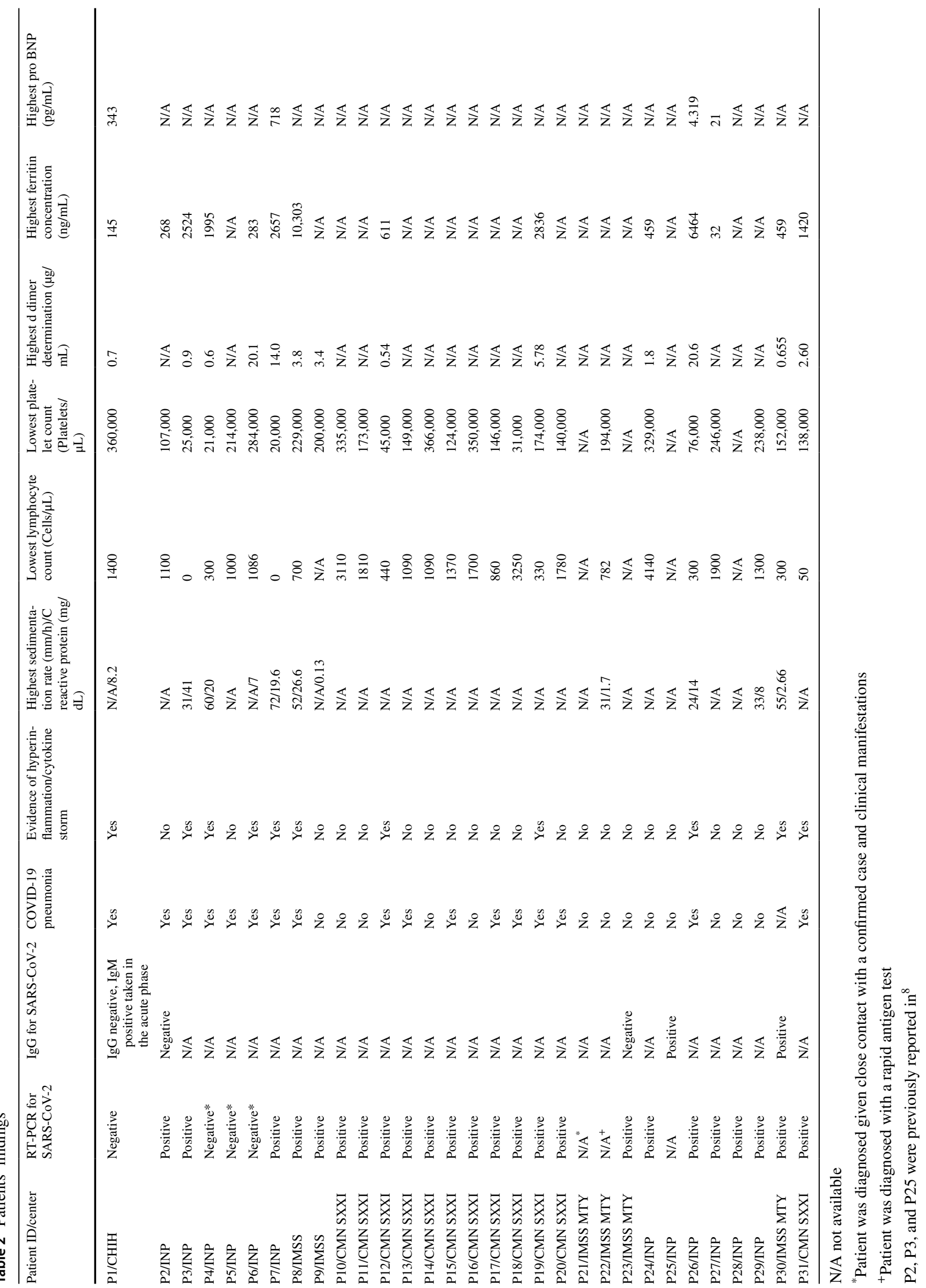


was no significant difference in patients who required hospital admission. Eleven patients had C-reactive protein (CRP) values measured (Reference value $<0.8 \mathrm{mg} / \mathrm{dL}$ [12]), 9 hospitalized and 2 ambulatory patients. We found higher values in patients who required hospital admission ( $14.0 \mathrm{vs} 0.9 \mathrm{mg} /$ $\mathrm{dL}$, Mann-Whitney U test $p=0.036$ ).

Complete blood counts (CBC) for lymphocyte and platelet counts were available in 26 patients, 11 ambulatory and 15 hospitalized. Lowest lymphocyte counts had a median of $1088(0-4,140)$ cells/uL. There was a lower median lymphocyte count in hospitalized patients (440 vs 1700 cells/uL, Mann-Whitney U test $p=0.004)$. Sixty-one percent of the patients had lymphopenia below 1500 cells/uL, and this finding was associated with higher odds of hospital admission (Fisher's exact test $p=0.021$, OR 16.8 95\%CI 1.6-176.2).

The median lowest platelet count was 152,000 (20,000-366,000) platelets/uL (Reference values 150,000-350,000). While thrombocytopenia was seen frequently in 12 out of 27 patients (44\%), it was usually not severe; only 5 patients had thrombocytopenia below 50,000 platelets/uL. The D-dimer value was available in 13 patients, only 1 ambulatory; median was $2.6(0.5-20.6) \mu \mathrm{g} / \mathrm{mL}$ (Reference values $0.22-0.50 \mu \mathrm{g} / \mathrm{mL}$ ). We found no difference in $\mathrm{D}$-dimer values in patients requiring ICU admission.

Ferritin concentration was available in 14 patients, all hospitalized; the median highest ferritin value was 1015 $(32-10,303) \mathrm{ng} / \mathrm{mL}$ (Reference values $24-336 \mathrm{ng} / \mathrm{mL}$ in males and 11-307 $\mathrm{ng} / \mathrm{ml}$ in females). Hyperferritinemia over $500 \mathrm{ng} / \mathrm{dL}$ was associated with ICU admission (Fisher's exact test $p=0.026$, OR 35 95\%CI 1.7-703.0).

We had six deaths in our series: 4 children and 2 adults. Three of the pediatric patients died due to uncontrolled hyperinflammation unleashed by SARS-CoV-2, and one pediatric patient died from uncontrolled cytomegalovirus (CMV) infection. The two adult patients died from healthcare-associated infections from the same bacteria species (see Table 1).

For COVID-19 treatment, over $80 \%$ received treatment with immunoglobulin, either intravenous or subcutaneous. The most frequently used medications after immunoglobulin were macrolides (39\%) and corticosteroids (29\%). Two patients received cyclosporine A as part of an immunomodulatory regimen ( $\mathrm{P} 4$ and $\mathrm{P} 7)$. Only one patient received treatment with tocilizumab and ivermectin $(\mathrm{P} 4)$. One patient (P30) was under chronic treatment with anakinra for his IEI.

Four cases of interest: catastrophic hyperinflammation and a SCID patient.

Patient 7 was a patient with XLA (BTK) and chronic lung disease due to recurrent respiratory infections. He coursed with SARS-CoV-2 pneumonia that unleashed a secondary hemophagocytic syndrome, requiring treatment with IVIG, high-dose corticosteroids, cyclosporine A, and etoposide.
Table 3 Summary of patients' characteristics

\begin{tabular}{ll}
\hline Age (Median, range) & 17 years $(0.5-56)$ \\
\hline Male to female ratio & $2.9: 1$ \\
Hospital admission & $15 / 31(48 \%)$ \\
$\quad$ Combined immunodeficiencies $(n=3)$ & $3 / 3(100 \%)$ \\
$\quad$ Antibody defects $(n=20)$ & $7 / 20(35 \%)$ \\
Phagocytic defects $(n=5)$ & $2 / 5(40 \%)$ \\
ICU admission & $8 / 31(26 \%)$ \\
Combined immunodeficiencies $(n=3)$ & $1 / 3(33 \%)$ \\
Antibody defects $(n=20)$ & $5 / 20(25 \%)$ \\
Phagocytic defects $(n=5)$ & $1 / 5(20 \%)$ \\
Deaths & $6 / 31(19 \%)$ \\
Combined immunodeficiencies $(n=3)$ & $1 / 3(33 \%)$ \\
Antibody defects $(n=20)$ & $2 / 20(10 \%)$ \\
Phagocytic defects $(n=5)$ & $1 / 5(20 \%)$ \\
Hyperinflammation & $11 / 31(36 \%)$ \\
Combined immunodeficiencies $(n=3)$ & $2 / 3(67 \%)$ \\
Antibody defects $(n=20)$ & $6 / 20(30 \%)$ \\
Phagocytic defects $(n=5)$ & $1 / 5(20 \%)$ \\
Pneumonia & $17 / 31(55 \%)$ \\
Combined immunodeficiencies $(n=3)$ & $3 / 3(100 \%)$ \\
Antibody defects $(n=20)$ & $12 / 20(60 \%)$ \\
Phagocytic defects $(n=5)$ & $1 / 5(20 \%)$ \\
\hline
\end{tabular}

*Detailed data is available for combined immunodeficiencies, antibody, and phagocytic defects, since they were the most frequently encountered. Dysregulation disorders, autoinflammatory, and phenocopies were not included since only one patient from each was available and information can be found in Tables 1 and 2

The patient remained with pancytopenia despite treatment and ultimately perished due to pulmonary hemorrhage.

Patient 26 was an adolescent male with X-linked chronic granulomatous disease $(C Y B B)$ and recurrent infections, chronic vertebrae osteomyelitis, and suspected active lung tuberculosis, who presented with fever and rapidly progressed to cardiogenic shock, myocarditis, third grade auriculoventricular block, and multiorgan failure. He fulfilled MIS-C criteria with high-grade fever, elevated acute phase reactants, and severe multiorgan disease, including cardiac, renal, hepatic, lung, and hematologic compromise. He also had highly elevated acute-phase reactants, lymphopenia, thrombocytopenia, hyperferritinemia, and elevated NT-proBNP. Several family members were positive for SARS-CoV-2, as was the patient. Despite treatment with corticosteroids, IVIG and multiple vasoactive medications, pump failure continued, and the patient died.

Patient 30 was a 12-year-old male with an autoinflammatory disorder (recurrent fever, arthralgias, and high acute phase reactants, without a conclusive genetic diagnosis after whole-exome sequencing), who was chronically treated with anakinra, steroid, and IVIG. He had a catastrophic disease over $48 \mathrm{~h}$, starting with arthralgia and stupor, then rapidly 
Table 4 Hospital and ICU admission and mortality in children and adults

\begin{tabular}{lllll}
\hline & Children & Adults & Fisher's exact test & Odds ratio (95\%CI) \\
\hline Hospital admission & $11 / 16(69 \%)$ & $4 / 15(26 \%)$ & $p=0.032$ & $6.1(1.3-28.7)$ \\
ICU admission & $5 / 16(31 \%)$ & $3 / 15(20 \%)$ & $p=0.685$ & $1.8(0.4-9.5)$ \\
Mortality & $4 / 16(25 \%)$ & $2 / 15(13 \%)$ & $p=0.654$ & $2.2(0.3-14.1)$ \\
\hline
\end{tabular}

developing fever and systemic inflammation, ultimately causing his demise.

Patient 1 was a 5-month-old male infant of Mennonite descent from Northern Mexico, whose sister had died with pneumonia and candidiasis. A female cousin was diagnosed with SCID due to CD3d deficiency. He was admitted with respiratory distress and acrocyanosis for suspected COVID19 pneumonia, from which his mother was recovering. A chest CT scan revealed atypical pneumonia. The respiratory virus panel, including SARS-CoV-2, came back negative, but an antibody test taken during his second hospital day was IgM positive (IgG negative). Immunological workup reported pan-hypogammaglobulinemia and low $\mathrm{CD} 3+(\mathrm{CD} 4+, \mathrm{CD} 8+) \mathrm{T}$ cell counts. He had elevated serum CRP, D-dimer, CK-MB and NT-proBNP, with normal troponin. Liver function tests reported AST $52 \mathrm{UI} / \mathrm{L}$, ALT 22 UI/L, bilirubin $1.88 \mathrm{mg} / \mathrm{dL}$, albumin $3.2 \mathrm{~g} / \mathrm{dL}$, and procalcitonin $0.20 \mathrm{ng} / \mathrm{mL}$. His ferritin serum levels were $145 \mathrm{ng} / \mathrm{mL}$, IL-6: $1.40 \mathrm{pg} / \mathrm{mL}$, and LDH $555 \mathrm{UI} / \mathrm{L}$. Mild viral myocarditis was diagnosed based on elevated cardiac markers; the echocardiographic evaluation showed normal biventricular function, and the patient did not require aminergic support but was started on digoxin. He responded well to oxygen support, antibiotics, and IVIG at $2 \mathrm{~g} / \mathrm{kg}$ and was discharged home after days. Targeted exome sequencing for 407 genes identified a homozygous nonsense variant (p.Arg68Ter) in $C D 3 D$. The patient is currently alive and well, awaiting hematopoietic stem cell transplantation.

\section{Discussion}

We describe the findings and outcomes of 31 patients with COVID-19 in the context of different IEI during the first 12 months of the SARS-CoV-2 pandemic in Mexico. Of the patients $74 \%$ were male, $65 \%$ had antibody deficiencies, $48 \%$ were hospitalized, and $81 \%$ survived.

Ambulatory patients rarely have an extensive laboratory work-up taken, and some laboratory values should be cautiously interpreted as predictors. We did find higher CRP values in patients who required hospital admission and hyperferritinemia over $500 \mathrm{ng} / \mathrm{mL}$ more frequently in patients who required ICU admittance.

The limitations of this study include its retrospective nature and a relatively small sample. It is, however, the largest report from our country, with adult and pediatric patients from four centers in three cities.

Around the world, there are currently reports of over 330 patients with known IEI who suffered from SARS-CoV-2 infection. More than $80 \%$ have survived [10, 13-16]. Three patients from this series, patients 2,3 and 25 , had been previously described in an international collaborative report [10]. Some case series have found a minor impact with a mild course among these patients [14], while others warn about higher rates of complications, care needs, and inpatient mortality rate [15]. In our series, pediatric patients with IEI had a higher hospital admission rate than adults. However, we found no difference in the frequency of pneumonia or hyperinflammation response. ICU admission and mortality were not significantly different in pediatric patients.

In patients with antibody defects, there have been reports suggesting that there is an inverse relationship between the severity of primary antibody deficiencies and the severity of SARS-CoV-2 infection, as some patients with agammaglobulinemia and absent B cells had a milder course when compared with patients with CVID, who have dysfunctional but present B cells [14]. This intrinsic lack of B cells might contribute to an attenuated inflammatory reaction to SARSCoV-2[17]. Several patients who recovered from COVID-19 lacked neutralizing antibodies[18, 19]. The relatively good outcome in patients with predominantly antibody defects would ratify the importance of cellular immunity against SARS-CoV-2. Nevertheless, it is not a simple matter, and we have not found a "rule of thumb" that stands without notable exceptions. No group of IEI seems to be immune or doomed to develop inflammatory complications.

Based on what we know about the pathophysiology of COVID-19 and the risk factors for severe disease, most of the damage is caused by an excessive, inappropriate immune response to the viral infection, not by the virus itself. Older age, obesity, and male sex are the strongest risk factors for severity and death in the general population. Besides immunosenescence and "inflammaging," any pro-inflammatory condition or immunosuppression could negatively affect the host response and outcome during SARS-CoV-2 infection $[3,4,15]$. Viral-induced type II and IV hypersensitivity reactions have been described in association with SARS-CoV-2. An overtly active immune response in COVID-19 might unbridle a disproportionately high inflammatory response $[19,20]$. 
When investigating patients with life-threatening COVID19; however, only autoantibodies against type 1 interferons and monogenic defects that involve viral sensing or interferon response have been identified, as opposed to a wider range of immune deficiencies [5, 6]. This holds true also for rheumatic conditions. The novel betacoronavirus infection does not seem to be more frequent or severe in patients with known systemic autoimmune diseases (not even in those who received rituximab) [21, 22], in HIV-coinfection under antiretroviral treatment, or in primary immunodeficient patients [10, 23, 24].

Patients with inflammasome-mediated autoinflammatory disorders deserve special consideration, as the NLRP3 inflammasome has been implicated in the development of uncontrolled systemic inflammatory response $[25,26]$. Those patients followed for cryopyrin-associated periodic syndrome, or any patients with inflammasome dysregulation, should probably take extreme preventive measures to avoid exposure.

SARS-CoV-2 RT-PCR detects viral RNA; and while a positive result is highly specific for the presence of the virus, the sensitivity is not well determined and can be affected by the timing of testing, sample collection, and laboratory techniques [27]. In our series, $75 \%$ had a positive result of the RT-PCR test. Out of the seven negative patients, two were confirmed through specific antibodies, one with a rapid colorimetric test, and the other four were considered infected given their caregivers' positive results and the patients' symptoms. Thus, a negative RT-PCR does not exclude COVID-19 infection.

When analyzing the cause of death of our patients, we noticed that none were directly attributable to the virus. Two adult patients died from healthcare-associated infections from the same bacteria species (Pseudomonas aeruginosa and Acinetobacter baumannii). It is important to bear in mind that patients with IEI might have a higher susceptibility to nosocomial infections [15], and when hospitalized for any cause, protective measures should be kept in place, as immunocompromised patients might have higher rates of morbidity and mortality from secondary bacterial infections.

Some patients with COVID-19 might develop a hyperinflammation phenotype, which might contribute to morbidity and mortality[28]. Hyperinflammation associated with SARS-CoV-2 infection was seen in 11 patients, 4 of them adults. A subset of patients with COVID-19 and hyperinflammation may present with a multisystemic inflammatory syndrome (MIS), with symptoms overlapping with Kawasaki disease and frequently showing signs of cardiac dysfunction [29-31]. MIS-C was originally described in pediatric patients, but a similar multisystem inflammatory syndrome in adults (MIS-A) has been described with edematous myocarditis, frequently requiring intensive care and aminergic support [32-34]. Both adult patients who died in our series had evidence of hyperinflammation, but none had evidence of MIS-A.

Three of the pediatric patients perished from uncontrolled hyperinflammation, two from MIS-C and one from secondary hemophagocytic lymphohistiocytosis (HLH). Patient 30 had an autoinflammatory disorder, P26 had CGD, and P7 had XLA. Underlying IEI might have predisposed them to undergo a "perfect (cytokine) storm," despite timely treatment with IVIG [35]. HLH has been recognized as an important complication of CGD, and COVID-19 may be a trigger [36, 37]. Meyts et al. reported one CGD patient with COVID-19 complicated with HLH associated to Burkholderia, with three other CGD patients presenting a mild disease [10]. Our deceased patient with CGD (P26) presented a clinical picture compatible with MIS-C refractory to IVIG and corticosteroids.

SARS-CoV-2 infection might worsen the residual immune function of patients living with IEI. Patient 3 had a severe systemic reactivation of CMV, progressive and refractory, which was triggered after COVID-19, despite having had several negative viral loads before; this ultimately caused his death. We also noted that patients with IEI and those who undergo transplantation might have a prolonged SARS-CoV-2 excretion over 6-8 weeks, as has been seen with other viral respiratory infections [38, 39].

SARS-CoV-2 infection can also trigger autoimmunity in predisposed patients [40-42]; in patient 24, the infection seems to have unleashed an exacerbation of autoimmune hemolytic anemia in a patient who had been controlled with immunomodulatory treatment. Interestingly, this patient did not present any respiratory manifestations of COVID-19.

Finally, we might be observing a protective effect of preexisting immunological conditions in patients who seek medical attention for COVID-19. Healthcare providers are more likely to be aware of their IEI patients' susceptibility of infections and risk of non-infectious complications, more prone to admit to a hospital under close supervision, to start invasive procedures and second-line treatments, and to ask for help. A "VIP" treatment of sorts, for our most vulnerable patients.

Over $80 \%$ of our patients received IVIG as treatment during the acute infection. Human IVIG preparations might have antibodies derived from common cold coronaviruses, which could cross-react with SARS-CoV-2 and have an immunomodulatory effect $[43,44]$. The timing of treatment with immune suppressants is crucial: dexamethasone, tocilizumab, or anakinra should only be started when the patient is admitted for COVID-19 pneumonia or if there is evidence of hyperinflammation. Immune modulation may be considered to blunt the excessive immune response induced by the virus [20, 45-49]. In contrast, interferon treatment may be a suitable therapeutic option early when the patient becomes symptomatic [50, 51]. Early type I interferon administration 
might also be beneficial in selected patients with IEI or auto antibodies impairing type I interferon response [52, 53].

In the future, we would like to collect and compare more information from Mexican patients and reports from around the world, to confirm associations and further explore potential risk and protective factors.

In conclusion, most of our known patients with IEI who acquired the SARS-CoV-2 infection developed a well-tolerated infection and survived. Continued, early, and high-dose treatment with IVIG might be beneficial in this group of patients. Pediatric patients with IEI may have a higher need for hospitalization. An impaired immune response due to IEI may be a predisposing factor for some and, paradoxically, a protective factor for others. COVID-19 may be the first in a series of unfortunate events that lead to a patient's demise, either due to hyperinflammation or deterioration of residual immune function; healthcare-associated infections should be aggressively prevented.

Abbreviations $C G D$, Chronic granulomatous disease; $C M V$, Cytomegalovirus; COVID-19, Coronavirus disease 2019; CVID, Common-variable immune deficiency; HSTC, Hematopoietic stem cell transplantation; IBM, International Business Machines Corporation; IVIG, Intravenous immunoglobulin; $M I S$ - $C$, Multisystem inflammatory syndrome in children; $M I S-A$, Multisystem inflammatory syndrome in adults; $R T-P C R$, Real-time polymerase chain reaction; $S A D$, Specific antibody deficiency; SARS-CoV-2, Severe Acute Respiratory Syndrome Coronavirus 2; $S C I D$, Severe combined immune deficiency; WAS, Wiskott-Aldrich syndrome; XLA, X-linked Agammaglobulinemia

Acknowledgements We are grateful to Dr Bertrand Boisson at the St Giles Laboratory of Human Genetics of Infectious Diseases, Rockefeller University, New York, NY, who helped us diagnose the patient with ARPC1B deficiency through whole-exome sequencing analysis. Dr Daniel Kastner and his team at the NIH, Bethesda, investigated and treated the patient with autoinflammation. The Jeffrey-Modell Foundation paid for the Invitae NGS 407 gene panel for Patient 1. Fundación Mexicana para Niñ@s con Inmunodeficiencias primarias (FUMENI, AC) facilitates our work by covering the costs of patient samples shipping when needed. We also thank all primary care physicians for taking care of patients with inborn errors of immunity and other rare diseases in this difficult time.

Authors' contributions Substantial contribution to the acquisition of the data were provided by L.M. Castano-Jaramillo, M.A. YamazakiNakashimada, P.M. O'Farrill-Romanillos, D. Muzquiz Zermeño, S.C. Scheffler Mendoza, E. Venegas Montoya, J.A. García Campos, L.M. Sánchez-Sánchez, L.B. Gámez González, J.M. Ramírez López, J.C Bustamante Ogando, E. Vásquez-Echeverri, E.A. Medina Torres, G. Lopez-Herrera, L. Blancas Galicia, L. Berrón Ruiz, A.T StainesBoone, S.E. Espinosa-Padilla, N.H. Segura Mendez, and S.O. Lugo Reyes. Substantial contribution to the conception of the work, analysis of the data, and drafting the manuscript were provided by L.M. Castano-Jaramillo and S.O. Lugo Reyes. Yamazaki-Nakashimada, P.M. O'Farrill-Romanillos, D. Muzquiz Zermeño, S.C. Scheffler Mendoza, E. Venegas Montoya, J.A. García Campos, L.M. Sánchez-Sánchez, L.B. Gámez González, J.M. Ramírez López, J.C Bustamante Ogando, E. Vásquez-Echeverri, E.A. Medina Torres, G. Lopez-Herrera, L. Blancas Galicia, L. Berrón Ruiz, A.T Staines-Boone, S.E. Espinosa-Padilla, and N.H. Segura Mendez critically revised the manuscript. All authors read and approved the final manuscript.

Funding No funding was received for this manuscript.

Data availability All data generated or analyzed during this study are included in this published article.

\section{Declarations}

Ethics approval The study was approved, as part of a larger project, by the Research and Ethics committees at the National Institute of Pediatrics.

Consent to participate and to publish. Written informed consent was obtained from all patients or their legal guardians.

Competing interests We have no competing interests to disclose.

\section{References}

1. Coronavirus Update (Live): $114,530,163$ Cases and 2,539,955 Deaths from COVID-19 Virus Pandemic - Worldometer [Internet]. [cited $2021 \mathrm{Feb} 28$ ]. Available from: https://www.world ometers.info/coronavirus/

2. Zhou F, Yu T, Du R, Fan G, Liu Y, Liu Z, et al. Clinical course and risk factors for mortality of adult inpatients with COVID-19 in Wuhan, China: a retrospective cohort study. The Lancet Elsevier. 2020;395:1054-62.

3. Gandhi RT, Lynch JB, Del Rio C. Mild or Moderate Covid-19. N Engl J Med. 2020;383:1757-66.

4. Pietrobon AJ, Teixeira FME, Sato MN. I mmunosenescence and Inflammaging: Risk Factors of Severe COVID-19 in Older People. Front Immunol. 2020;11:579220.

5. Zhang Q, Bastard P, Liu Z, Le Pen J, Moncada-Velez M, Chen J, et al. Inborn errors of type I IFN immunity in patients with lifethreatening COVID-19. Science. 2020;370.

6. Bastard P, Rosen LB, Zhang Q, Michailidis E, Hoffmann H-H, Zhang Y, et al. Autoantibodies against type I IFNs in patients with life-threatening COVID-19. Science [Internet]. American Association for the Advancement of Science; 2020 [cited 2021 Apr 21];370. Available from: https://science.sciencemag.org/content/ 370/6515/eabd4585

7. Conti P, Ronconi G, Caraffa A, Gallenga C, Ross R, Frydas I, et al. Induction of pro-inflammatory cytokines (IL-1 and IL-6) and lung inflammation by Coronavirus-19 (COVI-19 or SARSCoV-2): anti-inflammatory strategies. J Biol Regul Homeost Agents. 2020;34:327-31.

8. Hadjadj J, Yatim N, Barnabei L, Corneau A, Boussier J, Smith N, et al. Impaired type I interferon activity and inflammatory responses in severe COVID-19 patients. Science. 2020;369:718-24.

9. Tangye SG, Al-Herz W, Bousfiha A, Chatila T, CunninghamRundles C, Etzioni A, et al. Human Inborn Errors of Immunity: 2019 Update on the Classification from the International Union of Immunological Societies Expert Committee. J Clin Immunol. 2020;40:24-64.

10. Meyts I, Bucciol G, Quinti I, Neven B, Fischer A, Seoane E, et al. Coronavirus disease 2019 in patients with inborn errors of immunity: An international study. J Allergy Clin Immunol. 2021;147:520-31. 
11. Sox HC, Liang MH. The erythrocyte sedimentation rate. Guidelines for rational use. Ann Intern Med. 1986;104:515-23.

12. Mayo Clinic Laboratories [Internet]. [cited 2021 Apr 21]. Available from: https://www.mayocliniclabs.com/index.html

13. Marcus N, Frizinsky S, Hagin D, Ovadia A, Hanna S, Farkash M, et al. Minor clinical impact of COVID-19 Pandemic on patients with primary immunodeficiency in Israel. Front Immunol. 2020;11:614086.

14. Quinti I, Lougaris V, Milito C, Cinetto F, Pecoraro A, Mezzaroma I, et al. A possible role for B cells in COVID-19? Lesson from patients with agammaglobulinemia. J Allergy Clin Immunol. 2020;146:211-213.e4.

15. Delavari S, Abolhassani H, Abolnezhadian F, Babaha F, Iranparast $\mathrm{S}$, Ahanchian $\mathrm{H}$, et al. Impact of SARS-CoV-2 pandemic on patients with primary immunodeficiency. J Clin Immunol. 2021;41:345-55.

16. Castano-Jaramillo LM, Yamazaki-Nakashimada MA, Scheffler Mendoza SC, Bustamante-Ogando JC, Espinosa-Padilla SE, Lugo Reyes SO. A male infant with COVID-19 in the context of ARPC1B deficiency. Pediatr Allergy Immunol. 2020;

17. Babaha F, Rezaei N. Primary immunodeficiency diseases in COVID-19 Pandemic: a predisposing or protective factor? Am J Med Sci [Internet]. 2020 [cited 2020 Oct 7]; Available from: https://www.ncbi.nlm.nih.gov/pmc/articles/PMC7388814/

18. Wu F, Wang A, Liu M, Wang Q, Chen J, Xia S, et al. Neutralizing antibody responses to SARS-CoV-2 in a COVID-19 recovered patient cohort and their implications. medRxiv. Cold Spring Harbor Laboratory Press; 2020;2020.03.30.20047365.

19. Corse T, Dayan L, Kersten S, Battaglia F, Terlecky SR, Han Z. Clinical outcomes of covid-19 patients with pre-existing, compromised immune systems: a review of case reports. Int J Med Sci. 2020;17:2974-86.

20. Icenogle T. COVID-19: Infection or Autoimmunity. Front Immunol [Internet]. Frontiers; 2020 [cited $2021 \mathrm{Feb} 28$ ];11. Available from: https://www.frontiersin.org/articles/https://doi.org/10.3389/ fimmu.2020.02055/full

21. Baker D, Roberts CAK, Pryce G, Kang AS, Marta M, Reyes $\mathrm{S}$, et al. COVID-19 vaccine-readiness for anti-CD20-depleting therapy in autoimmune diseases. Clin Exp Immunol. 2020;202:149-61.

22. Batu ED, Özen S. Implications of COVID-19 in pediatric rheumatology. Rheumatol Int. 2020;40:1193-213.

23. Xu Z, Zhang C, Wang FS. COVID-19 in people with HIV. Lancet HIV. 2020;7:e524-6.

24. Castel AD, Wilbourn B, Magnus M, Greenberg AE. SARS-CoV-2 and HIV: Epidemiology, Treatment, and Lessons Learned from HIV. AIDS Rev. 2020;22:133-42.

25. Freeman TL, Swartz TH. Targeting the NLRP3 Inflammasome in Severe COVID-19. Front Immunol [Internet]. 2020 [cited 2021 Feb 28];11. Available from: https://www.ncbi.nlm.nih.gov/pmc/ articles/PMC7324760/

26. van den Berg DF, te Velde AA. Severe COVID-19: NLRP3 Inflammasome Dysregulated. Front Immunol [Internet]. 2020 [cited $2021 \mathrm{Feb} 28$ ];11. Available from: https://www.ncbi.nlm. nih.gov/pmc/articles/PMC7332883/

27. RT-PCR Testing [Internet]. [cited $2021 \mathrm{Feb} 20$ ]. Available from: https://www.idsociety.org/covid-19-real-time-learning-network/ diagnostics/RT-pcr-testing/

28. Manson JJ, Crooks C, Naja M, Ledlie A, Goulden B, Liddle T, et al. COVID-19-associated hyperinflammation and escalation of patient care: a retrospective longitudinal cohort study. The Lancet Rheumatology Elsevier. 2020;2:e594-602.

29. Whittaker E, Bamford A, Kenny J, Kaforou M, Jones CE, Shah $\mathrm{P}$, et al. Clinical characteristics of 58 children with a pediatric inflammatory multisystem syndrome temporally associated with SARS-CoV-2. JAMA. 2020;
30. Belhadjer Z, Méot M, Bajolle F, Khraiche D, Legendre A, Abakka $S$, et al. Acute heart failure in multisystem inflammatory syndrome in children (MIS-C) in the context of global SARS-CoV-2 pandemic. Circulation. 2020;

31. Riphagen S, Gomez X, Gonzalez-Martinez C, Wilkinson N, Theocharis P. Hyperinflammatory shock in children during COVID-19 pandemic. Lancet. 2020;395:1607-8.

32. Sokolovsky S, Soni P, Hoffman T, Kahn P, Scheers-Masters J. COVID-19 associated Kawasaki-like multisystem inflammatory disease in an adult. Am J Emerg Med. 2021;39:253.e1-253.e2.

33. Tenforde MW, Morris SB. Multisystem inflammatory syndrome in adults: coming into focus. CHEST Elsevier. 2021;159:471-2.

34. Hékimian G, Kerneis M, Zeitouni M, Cohen-Aubart F, Chommeloux J, Bréchot N, et al. Coronavirus Disease 2019 Acute myocarditis and multisystem inflammatory syndrome in adult intensive and cardiac care units. CHEST Elsevier. 2021;159:657-62.

35. Rieber N, Hector A, Kuijpers T, Roos D, Hartl D. Current concepts of hyperinflammation in chronic granulomatous disease. Clin Dev Immunol. 2012;2012:252460.

36. Álvarez-Cardona A, Rodríguez-Lozano AL, Blancas-Galicia L, Rivas-Larrauri FE, Yamazaki-Nakashimada MA. Intravenous immunoglobulin treatment for macrophage activation syndrome complicating chronic granulomatous disease. J Clin Immunol. 2012;32:207-11.

37. Blancas-Galicia L, Santos-Chávez E, Deswarte C, Mignac Q, Medina-Vera I, León-Lara X, et al. Genetic, Immunological, and Clinical Features of the First Mexican Cohort of Patients with Chronic Granulomatous Disease. J Clin Immunol. 2020;40:475-93.

38. van der Vries E, Stittelaar KJ, van Amerongen G, Veldhuis Kroeze EJB, de Waal L, Fraaij PLA, et al. Prolonged influenza virus shedding and emergence of antiviral resistance in immunocompromised patients and ferrets. PLoS Pathog [Internet]. 2013 [cited 2020 Oct 7];9. Available from: https://www.ncbi.nlm.nih.gov/ pmc/articles/PMC3662664/

39. Dueñas JAC. Prolonged viral shedding in an immunocompromised pediatric patient with influenza A H1N1 (2009): a case report and literature review. Alerg Asma Inmunol Pediatr Medigraphic. 2015;24:91-4.

40. Ehrenfeld M, Tincani A, Andreoli L, Cattalini M, Greenbaum A, Kanduc D, et al. Covid-19 and autoimmunity. Autoimmunity Reviews. Elsevier; 2020;19:102597.

41. Ciaffi J, Meliconi R, Ruscitti P, Berardicurti O, Giacomelli R, Ursini F. Rheumatic manifestations of COVID-19: a systematic review and meta-analysis. BMC Rheumatology. 2020;4:65.

42. Liu Y, Sawalha AH, Lu Q. COVID-19 and autoimmune diseases. Curr Opin Rheumatol. 2021;33:155-62.

43. Soresina A, Moratto D, Chiarini M, Paolillo C, Baresi G, Focà E, et al. Two X-linked agammaglobulinemia patients develop pneumonia as COVID-19 manifestation but recover. Pediatr Allergy Immunol. 2020;

44. Liu BM, Hill HR. Role of host immune and inflammatory responses in COVID-19 cases with underlying primary immunodeficiency: a review. J Interferon Cytokine Res. 2020;40:549-54.

45. Cavalli G, Larcher A, Tomelleri A, Campochiaro C, Della-Torre E, Luca GD, et al. Interleukin-1 and interleukin-6 inhibition compared with standard management in patients with COVID-19 and hyperinflammation: a cohort study. The Lancet Rheumatology [Internet]. Elsevier; 2021 [cited 2021 Feb 28];0. Available from: https://www.thelancet.com/journals/lanrhe/article/PIIS26659913(21)00012-6/abstract

46. REMAP-CAP Investigators, Gordon AC, Mouncey PR, AlBeidh F, Rowan KM, Nichol AD, et al. Interleukin-6 receptor antagonists in critically Ill patients with Covid-19. N Engl J Med. 2021;384:1491-502. 
47. Rosas IO, Bräu N, Waters M, Go RC, Hunter BD, Bhagani S, et al. Tocilizumab in hospitalized patients with severe Covid-19 pneumonia. N Engl J Med. 2021;384:1503-16.

48. Pontali E, Volpi S, Signori A, Antonucci G, Castellaneta M, Buzzi $D$, et al. Efficacy of early anti-inflammatory treatment with high doses IV Anakinra with or without glucocorticoids in patients with severe COVID-19 pneumonia. J Allergy Clin Immunol. 2021;147(4):1217-25.

49. The RECOVERY Collaborative Group. Dexamethasone in Hospitalized Patients with Covid-19. New England Journal of Medicine. Massachusetts Medical Society; 2021;384:693-704.

50. Haji Abdolvahab M, Moradi-kalbolandi S, Zarei M, Bose D, Majidzadeh-A K, Farahmand L. Potential role of interferons in treating COVID-19 patients. Int Immunopharmacol. 2021;90:107171.
51. Wang B, Li D, Liu T, Wang H, Luo F, Liu Y. Subcutaneous injection of IFN alpha-2b for COVID-19: an observational study. BMC Infect Dis. 2020;20:723.

52. Bastard P, Lévy R, Henriquez S, Bodemer C, Szwebel T-A, Casanova J-L. Interferon- $\beta$ therapy in a patient with incontinentia pigmenti and autoantibodies against type I IFNs infected with SARS-CoV-2. J Clin Immunol. 2021:1-3. https://doi.org/10.1007/ s10875-021-01023-5.

53. Lévy R, Bastard P, Lanternier F, Lecuit M, Zhang S-Y, Casanova J-L. IFN- $\alpha 2$ a Therapy in Two Patients with Inborn Errors of TLR3 and IRF3 Infected with SARS-CoV-2. J Clin Immunol. 2021;41:26-7.

Publisher's note Springer Nature remains neutral with regard to jurisdictional claims in published maps and institutional affiliations.

\section{Authors and Affiliations}

\section{Lina M. Castano-Jaramillo ${ }^{1} \cdot$ Marco Antonio Yamazaki-Nakashimada ${ }^{1}$ Patricia M. O'Farrill-Romanillos ${ }^{2}$. David Muzquiz Zermeño ${ }^{3}$. Selma C. Scheffler Mendoza ${ }^{1}$ - Edna Venegas Montoya ${ }^{3}$. Jorge Alberto García Campos ${ }^{4}$. Luz María Sánchez-Sánchez ${ }^{5}$. Luisa B. Gámez González ${ }^{6}$. Jesús Moisés Ramírez López ${ }^{7}$. Juan Carlos Bustamante Ogando ${ }^{8}$. Estefanía Vásquez-Echeverri ${ }^{8}$. Edgar Alejandro Medina Torres ${ }^{8}$. Gabriela Lopez-Herrera ${ }^{8} \cdot$ Lizbeth Blancas Galicia $^{8}$. Laura Berrón Ruiz ${ }^{8}$. Aidé Tamara Staines-Boone ${ }^{3}$. Sara Elva Espinosa-Padilla ${ }^{8} \cdot$ Nora Hilda Segura Mendez $^{2} \cdot$ Saul O. Lugo Reyes $^{8}$}

Saul O. Lugo Reyes

dr.lugo.reyes@gmail.com

1 Clinical Immunology Service, National Institute of Pediatrics, Mexico City, Mexico

2 Allergy and Immunology Department, Hospital de Especialidades, National Medical Center, Siglo XXI" IMSS, Mexico City, Mexico

3 Immunology Service, Hospital de Especialidades UMAE 25 IMSS, Monterrey, NL, Mexico

4 Infectious Disease, Hospital de Especialidades UMAE 25 IMSS, Monterrey, NL, Mexico
5 Pediatrics Departments, Hospital de Especialidades UMAE 25 IMSS, Monterrey, NL, Mexico

6 Clinical Immunology Service, Hospital Infantil Especialidades de Chihuahua, Chihuahua, Mexico

7 Infectious Diseases Service, Hospital Infantil Especialidades de Chihuahua, Chihuahua, Mexico

8 Immune Deficiencies Lab., National Institute of Pediatrics, Av Iman 1, Torre de Investigacion Piso 9, Insurgentes Cuicuilco, Coyoacan, 04530 Mexico City, Mexico 